\title{
Simultaneous Determination of Atractylenolide I and II in Rat Plasma by UPLC-MS/MS and Its Application to Pharmacokinetic Study after Intravenous Administration
}

\author{
Lianguo Chen ${ }^{1}$, Haiya $\mathrm{Wu}^{2}$, Xiaoting $\mathrm{Tu}^{3}$, Yi Zhao ${ }^{3}$, Yanyan Jiang ${ }^{3}$, Congcong $\mathrm{Wen}^{3^{*}}$ and Yue Luo ${ }^{2 *}$ \\ ${ }^{1}$ Department of Pharmacy, Wenzhou People's Hospital, Wenzhou, China \\ ${ }^{2}$ The Second Affiliated Hospital and Yuying Children's Hospital, Wenzhou Medical University, Wenzhou, China \\ ${ }^{3}$ Laboratory Animal Centre, Wenzhou Medical University, Wenzhou, China
}

Received: 11 March 2017; accepted: 01 October 2017

\begin{abstract}
Atractylodis exerted a variety of pharmacological effects such as anti-tumor, anti-inflammatory, anti-bacterial, and antiaging effects etc. The major ingredients of Atractylodis are atractylenolide I and II that exhibited activities in antiinflammatory and anticancer. In this work, a sensitive and selective ultra-performance liquid chromatography-tandem mass spectrometry (UPLC-MS/MS) method for determination of atractylenolide I and II in rat plasma was developed. The UPLC-MS/MS method was validated for selectivity, linearity, accuracy, precision, recovery, and stability with a total run time of $4.0 \mathrm{~min}$. After addition of atractylenolide III as an internal standard (IS), protein precipitation by acetonitrile was used to prepare samples. Chromatographic separation was achieved on a UPLC BEH C18 column $(2.1 \mathrm{~mm} \times$ $50 \mathrm{~mm}, 1.7 \mu \mathrm{m}$ ) with $0.1 \%$ formic acid and acetonitrile as the mobile phase with gradient elution. An electrospray ionization source was applied and operated in positive ion mode; multiple reactions monitoring (MRM) mode was used for quantification using target fragment ions $\mathrm{m} / \mathrm{z} 231.1 \rightarrow 185.1$ for atractylenolide I, $\mathrm{m} / z 233.1 \rightarrow 91.0$ for II, and $\mathrm{m} / \mathrm{z}$ $249.0 \rightarrow 231.1$ for IS. Calibration plots were linear throughout the range $1-1000 \mathrm{ng} / \mathrm{mL}$ for atractylenolide I and II in rat plasma. Mean recoveries of atractylenolide I and II in rat plasma ranged from $86.2 \%$ to $96.3 \%$. Relative standard deviation (RSD) of intra-day and inter-day precision was both less than $12 \%$. The accuracy of the method was between $91.0 \%$ and $109.0 \%$. The method was successfully applied to pharmacokinetic study of atractylenolide I and II after intravenous administration in rats.
\end{abstract}

Keywords: Atractylenolide I, atractylenolide II, UPLC-MS/MS, pharmacokinetics, plasma

\section{Introduction}

Atractylodis can cure patients with anorexia, excessive perspiration, splenic asthenia, and abnormal fetal movement. Atractylodis exerted a variety of pharmacological effects such as anti-inflammatory, anti-tumor, anti-bacterial, and anti-aging effects etc. [1-3]. The major ingredients of Atractylodis are atractylenolide I and II that exhibited activities in anti-inflammatory and anticancer [4-6]. Therefore, the pharmacokinetics of atractylenolide I and II should be researched for evaluating clinical applications of Atractylodis.

There have been several literatures reported for determination atractylenolide I or atractylenolide II in rat plasma [1, 7-10]. Yan et al. developed a simple and rapid ultra-performance liquid chromatography-tandem mass spectrometry (UPLC-MS/MS) method for the simultaneous determination of atractylenolide I, II, and III in rat plasma [1]. Plasma samples were processed by liquid-liquid extraction with ethyl acetate, using schisandrin as internal standard (IS). This method was successfully applied to the comparative pharmacokinetic study of atractylenolide I, II, and III in rat plasma after intragastric administration of Baizhufuling extract and Atractylodis extract. Shi et al. developed and validated a sensitive, rapid, and selective liquid chromatography-tandem mass spectrometry (LC-MS/MS) method for the simultaneous determination of atractylenolide II and atractylenolide III in rat plasma using loliolide as IS [8]. After protein precipitation with ethyl acetate, the analytes were injected into an LC-MS/MS system for quantification. The validated method was successfully applied to the pharmacokinetic

* Authors for correspondence: bluce494949@163.com, bassini@163.com study of atractylenolide II and atractylenolide III in rat plasma after oral administration of Atractylodis Macrocephala Rhizoma extract. However, the pharmacokinetic study of atractylenolide I or atractylenolide II after intravenous administration in rats was not reported.

In comparison with conventional analytical techniques, ultra-performance liquid chromatography coupled with tandem mass spectrometry (UPLC-MS/MS) is documented to possess improved sensitivity, selectivity, and specificity in quantitative determination of the active compound of herbal drug in biological samples [11-14]. Consequently, in the present study, a UPLC-MS/MS method was established for the determination of atractylenolide I and II in rat plasma samples and was successfully applied to the pharmacokinetic after intravenous administration in rats.

\section{Experimental}

Chemicals and Reagents. Atractylenolide I and II (purity $>98 \%$, Figure 1a and b) as well as atractylenolide III (IS, purity $>98 \%$, Figure 1c) were purchased from the Chengdu Mansite Pharmaceutical Co. Ltd. (Chengdu, China). LC-grade acetonitrile and methanol were purchased from Merck Company (Darmstadt, Germany). Ultra-pure water was prepared by Millipore Milli-Q purification system (Bedford, MA, USA). Rat blank plasma samples were supplied by drug-free rats (Laboratory Animal Center of Wenzhou Medical University).

Instrumentation and Conditions. A UPLC-MS/MS system with ACQUITY I-Class UPLC and a XEVO TQS-micro triple quadrupole mass spectrometer (Waters Corp., Milford, MA,

This is an open-access article distributed under the terms of the Creative Commons Attribution-NonCommercial 4.0 International License (https://creativecommons.org/licenses/by-nc/4.0/), which permits unrestricted use, distribution, and reproduction in any medium for non-commercial purposes, provided the original author and source are credited, a link to the CC License is provided, and changes - if any - are indicated. 
<smiles>C=C1CCC[C@]2(C)C=C3OC(=O)C(C)=C3C[C@H]12</smiles>

(A)<smiles>C=C1CCC[C@]2(C)CC3OC(=O)C(C)=C3C[C@H]12</smiles>

(B)<smiles>C=C1CCC[C@]2(C)C[C@]3(O)OC(=O)C(C)=C3C[C@H]12</smiles>

(C)

Figure 1. Chemical structure of atractylenolide I (A) and II (B); atractylenolide III (IS, C)

USA), equipped with an electrospray ionization (ESI) interface, was used to analyze the compounds. Masslynx 4.1 software (Waters Corp.) was used for data acquisition and instrument control.

Atractylenolide I, II, and III (IS) were separated using a UPLC BEH C18 column $(2.1 \mathrm{~mm} \times 50 \mathrm{~mm}, 1.7 \mu \mathrm{m}$, Waters, USA $)$ maintained at $40{ }^{\circ} \mathrm{C}$. The initial mobile phase consisted of acetonitrile and water (containing $0.1 \%$ formic acid) with gradient elution at a flow rate of $0.4 \mathrm{~mL} / \mathrm{min} ; 0-0.2 \mathrm{~min}$, acetonitrile $10 \%$; $0.2-1.5 \mathrm{~min}$, acetonitrile $10 \%-80 \% ; 1.5-2.0 \mathrm{~min}$, acetonitrile $80 \%$; $2.0-2.5 \mathrm{~min}$, acetonitrile $80 \%-10 \%$; and $2.5-4.0 \mathrm{~min}$, acetonitrile $10 \%$. The total run time of the analytes was $4.0 \mathrm{~min}$.

Nitrogen was used as the desolvation gas $(800 \mathrm{~L} / \mathrm{h})$ and cone gas $(50 \mathrm{~L} / \mathrm{h})$. Ion monitoring conditions were defined as capillary voltage of $2.3 \mathrm{kV}$, source temperature of $150{ }^{\circ} \mathrm{C}$, and desolvation temperature of $400{ }^{\circ} \mathrm{C}$. Multiple reaction monitoring (MRM) modes of $\mathrm{m} / \mathrm{z} 231.1 \rightarrow 185.1$ for atractylenolide I, $\mathrm{m} / \mathrm{z} 233.1 \rightarrow$ 91.1 for II, and $m / z 249.0 \rightarrow 231.1$ for atractylenolide III were utilized to conduct quantitative analysis with ESI interface in positive mode.

Calibration Standards and Quality Control Samples. The stock solutions of atractylenolide I and II $(1.0 \mathrm{mg} / \mathrm{mL})$ as well as atractylenolide III (IS) $(1.0 \mathrm{mg} / \mathrm{mL})$ were prepared in methanol-water (50:50). The $0.5 \mu \mathrm{g} / \mathrm{mL}$ working standard solution of the IS was prepared from the IS stock solution by dilution with methanol; working solutions for calibration and controls were prepared from stock solutions similarly, using methanol diluent. All of the solutions were stored at $4{ }^{\circ} \mathrm{C}$ and were brought to room temperature before use.

Atractylenolide I and II calibration standards were prepared by spiking blank rat plasma with appropriate amounts of the working solutions. Calibration plots were offset to range between 1 and $1000 \mathrm{ng} / \mathrm{mL}$ for atractylenolide I and II in rat plasma at 1, 5, 20, $100,200,500$, and $1000 \mathrm{ng} / \mathrm{mL}$, each by adding $5 \mu \mathrm{L}$ of the appropriate working solution to $50 \mu \mathrm{L}$ of blank rat plasma, followed by short vortex mixing. Quality-control (QC) samples were blank

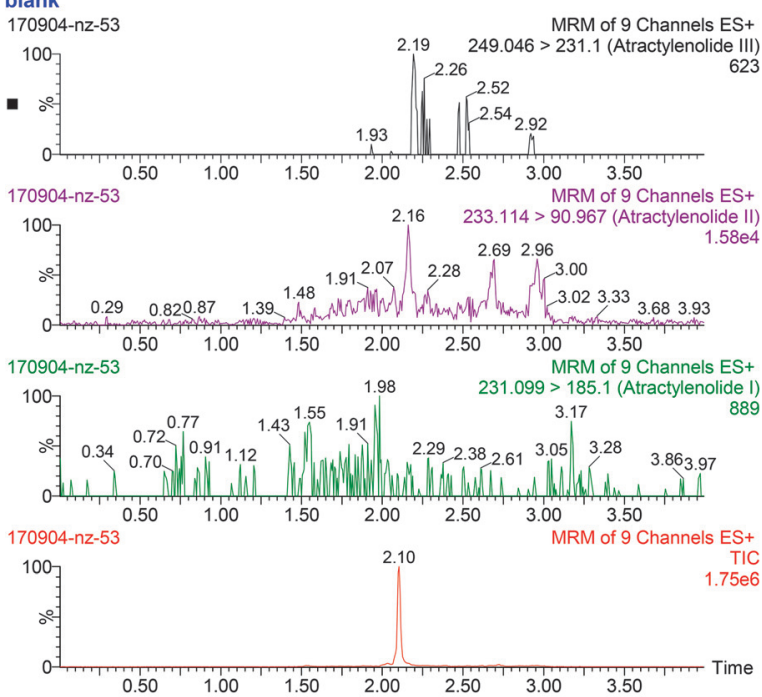

(A)

6
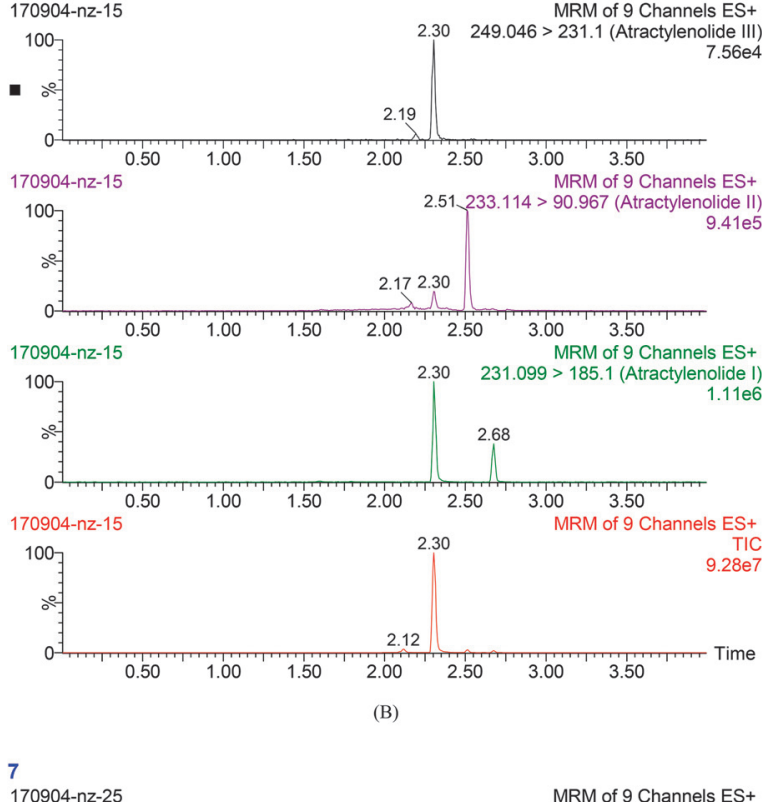
$\begin{array}{lll}\text { 170904-nz-25 } & \text { MRM of } 9 \text { Channels ES+ } \\ & 2.30 & 249.046>231.1\end{array}$
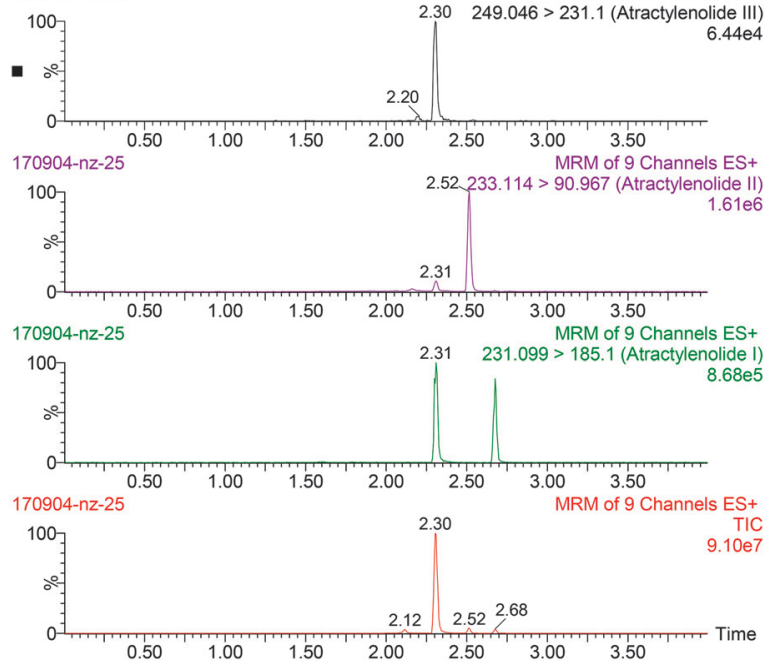

(C)

Figure 2. Representative UPLC-MS/MS chromatograms of atractylenolide I $\left(t_{\mathrm{R}}=2.68 \mathrm{~min}\right)$, atractylenolide II $\left(t_{\mathrm{R}}=2.52 \mathrm{~min}\right)$; atractylenolide III $\left(t_{R}=2.68 \mathrm{~min}\right.$, IS $)$. A, blank plasma spiked with IS; B, blank plasma spiked with atractylenolide I and II; IS; C, a rat plasma sample after intravenous administration of single dosage $5 \mathrm{mg} / \mathrm{kg}$ atractylenolide I and II 
prepared in the same manner as the calibration standards, in three different plasma concentrations $(2,180$, and $800 \mathrm{ng} / \mathrm{mL})$. The calibration standards and QC samples were prepared using protein precipitation by acetonitrile before UPLC-MS/MS analysis.

Sample Preparation. Before analysis, the plasma sample was thawed to room temperature. An aliquot of $5 \mu \mathrm{L}$ of the IS working solution $(0.5 \mu \mathrm{g} / \mathrm{mL})$ was added to $50 \mu \mathrm{L}$ of the collected plasma sample in a $1.5 \mathrm{~mL}$ centrifuge tube, followed by the addition of $150 \mu \mathrm{L}$ of acetonitrile. The tubes were vortex mixed for $1.0 \mathrm{~min}$. After centrifugation at $14,900 \mathrm{~g}$ for $10 \mathrm{~min}$, the supernatant $(2 \mu \mathrm{L})$ was injected into the UPLC-MS/MS system for analysis.

Method Validation. Rigorous tests for selectivity, linearity, accuracy, precision, recovery, and stability, according to the guidelines set by the United States Food and Drug Administration (FDA) and European Medicines Agency (EMA), were conducted in order to thoroughly validate the proposed bioanalytical method [15-20]. Validation runs were conducted on three consecutive days. Each validation run consisted of one set of calibration standards and six replicates of QC plasma samples.

Pharmacokinetic Study. Male Sprague-Dawley rats (200$220 \mathrm{~g}$ ) were obtained from the Laboratory Animal Center of Wenzhou Medical University to study the pharmacokinetics of atractylenolide I and II. All six rats were housed at the Laboratory Animal Center of Wenzhou Medical University. Diet was prohibited for $12 \mathrm{~h}$ before the experiment, but water was freely available. Blood samples $(0.2 \mathrm{~mL})$ were collected from the tail vein into heparinized $1.5 \mathrm{~mL}$ polythene tubes at $0.0833,0.25,0.5,1,2,4,6,8,12$, and $24 \mathrm{~h}$ after intravenous administration of atractylenolide I ( $5 \mathrm{mg} / \mathrm{kg})$ and II $(5 \mathrm{mg} / \mathrm{kg})$. The samples were immediately centrifuged at $3000 \mathrm{~g}$ for $10 \mathrm{~min}$. The plasma as-obtained was stored at $-20{ }^{\circ} \mathrm{C}$ until analysis. Plasma atractylenolide I and II concentration versus time data for each rat was analyzed by DAS (Drug and Statistics) software (version 2.0, Wenzhou Medical University).

\section{Results and Discussion}

Selectivity and Matrix Effect. Figure 2 showed typical chromatograms of a blank plasma sample; a blank plasma sample spiked with atractylenolide I, II, and IS; and a plasma sample. There were no interfering endogenous substances observed at the retention time of the atractylenolide $\mathrm{I}\left(t_{\mathrm{R}}=2.68 \mathrm{~min}\right)$, atractylenolide II $\left(t_{\mathrm{R}}=2.52 \mathrm{~min}\right)$, and atractylenolide III $\left(t_{\mathrm{R}}=2.68 \mathrm{~min}\right.$, IS $)$. The retention time of the atractylenolide II was not same as that reported in literatures, because of the different UPLC or HPLC conditions. Chromatographic separation was accomplished on a Thermo Hypersil GOLD C18 column $(2.1 \mathrm{~mm} \times 50 \mathrm{~mm}, 1.9 \mathrm{~mm})$ with mobile phase consisting of acetonitrile and 0.1\% formic acid-water (50:50, $\mathrm{v} / \mathrm{v}$ ) in work reported by Yan et al. [1]. Chromatography was performed using a Zorbax SB-C 18 column (2.1-50 mm, i.d. $1.8 \mathrm{~mm}$, Agilent Corporation, MA, USA), eluting with water and acetonitrile $(45: 55, \mathrm{v} / \mathrm{v})$ at $0.2 \mathrm{~mL} / \mathrm{min}$ in work reported by Shi et al. [8], while atractylenolide I, II, and III (IS) were separated using a UPLC BEH C18 column $(2.1 \mathrm{~mm} \times$ $50 \mathrm{~mm}, 1.7 \mu \mathrm{m})$ with mobile phase consisted of acetonitrile
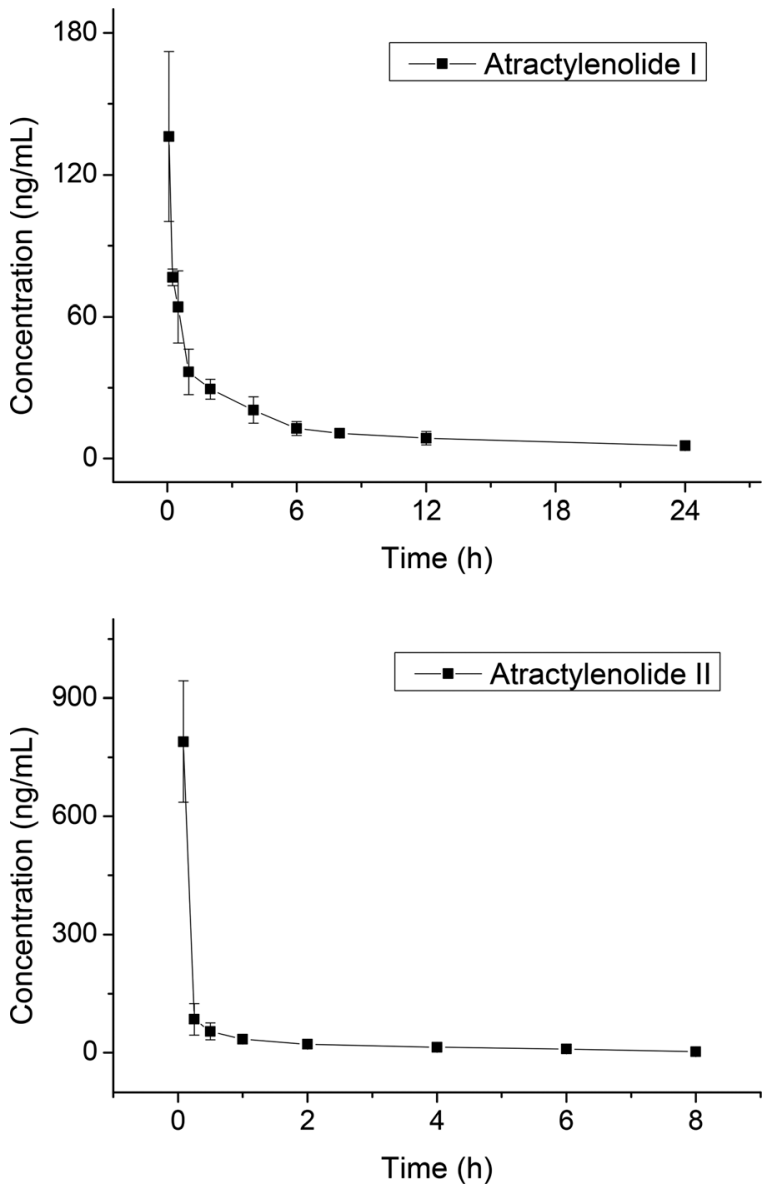

Figure 3. Mean plasma concentration time profile after intravenous $(5 \mathrm{mg} / \mathrm{kg})$ administration of atractylenolide I and II in rats

and water (containing $0.1 \%$ formic acid) with gradient elution at a flow rate of $0.4 \mathrm{~mL} / \mathrm{min}$ in our work.

The matrix effect for atractylenolide I and II at concentrations of 2, 180, and $800 \mathrm{ng} / \mathrm{mL}$ was measured between $90.3 \%$ and $102.6 \%(n=6)$. The matrix effect for IS $(50 \mathrm{ng} / \mathrm{mL})$ was $96.7 \%$ $(n=6)$. As a result, matrix effect from plasma was considered negligible in this method.

Calibration Curve and Sensitivity. Linear regressions of the peak area ratios versus concentrations were fitted over the concentration range $1-1000 \mathrm{ng} / \mathrm{mL}$ for atractylenolide I and II in rat plasma. The equation utilized to express the calibration curve is: $y=0.0000981007 x+0.000169991, r=0.9964$ for atractylenolide I and $y=0.000252421 x+0.000365777$,

Table 2. Primary pharmacokinetic parameters after intravenous administration of atractylenolide I and II in rats $(n=6)$

\begin{tabular}{lccc}
\hline Parameters & Unit & Atractylenolide I & Atractylenolide II \\
\hline $\mathrm{AUC}_{(0-t)}$ & $\mathrm{ng} / \mathrm{mL}^{*} \mathrm{~h}$ & $334.3 \pm 47.8$ & $277.0 \pm 38.8$ \\
$\mathrm{AUC}_{(0-\infty)}$ & $\mathrm{ng} / \mathrm{mL}^{*} \mathrm{~h}$ & $413.4 \pm 81.3$ & $285.3 \pm 36.8$ \\
$t_{1 / 2}$ & $\mathrm{~h}$ & $11.6 \pm 3.6$ & $1.9 \pm 1.0$ \\
$\mathrm{CL}$ & $\mathrm{L} / \mathrm{h} / \mathrm{kg}$ & $12.4 \pm 2.2$ & $17.7 \pm 2.3$ \\
$V$ & $\mathrm{~L} / \mathrm{kg}$ & $201.8 \pm 58.9$ & $48.5 \pm 26.6$ \\
$C_{\max }$ & $\mathrm{ng} / \mathrm{mL}$ & $136.2 \pm 35.9$ & $789.3 \pm 153.7$ \\
\hline
\end{tabular}

Table 1. Precision, accuracy, recovery; matrix effect for atractylenolide I and II of QC sample in rat plasma $(n=6)$

\begin{tabular}{|c|c|c|c|c|c|c|c|}
\hline \multirow[t]{2}{*}{ Compound } & \multirow[t]{2}{*}{ Concentration $(\mathrm{ng} / \mathrm{mL})$} & \multicolumn{2}{|c|}{ Precision (CV\%) } & \multicolumn{2}{|c|}{ Accuracy (\%) } & \multirow[t]{2}{*}{ Recovery } & \multirow[t]{2}{*}{ Matrix effect } \\
\hline & & Intra-day & Inter-day & Intra-day & Inter-day & & \\
\hline \multirow[t]{3}{*}{ Atractylenolide I } & 2 & 9.6 & 4.6 & 109.0 & 96.7 & 94.0 & 97.2 \\
\hline & 180 & 7.9 & 9.2 & 106.8 & 103.0 & 89.9 & 95.6 \\
\hline & 800 & 5.5 & 5.5 & 93.1 & 101.7 & 96.3 & 90.3 \\
\hline \multirow[t]{3}{*}{ Atractylenolide II } & 2 & 11.2 & 5.3 & 105.5 & 104.8 & 91.9 & 96.2 \\
\hline & 180 & 4.7 & 7.3 & 98.8 & 107.6 & 89.8 & 98.5 \\
\hline & 800 & 3.7 & 3.0 & 91.0 & 99.6 & 86.2 & 102.6 \\
\hline
\end{tabular}


$r=0.9979$ for atractylenolide II, where $y$ represents the ratios of atractylenolide I and II peak area to that of IS, and $x$ represents the plasma concentration. The lower limit of quantification (LLOQ) for the determination of atractylenolide I and II in plasma was $1 \mathrm{ng} / \mathrm{mL}$. The precision and accuracy at LLOQ were $14.5 \%$ and $87.6 \%$, respectively. The LOD, defined as a signal/noise ratio of 3 , was $0.5 \mathrm{ng} / \mathrm{mL}$ for atractylenolide I and $0.3 \mathrm{ng} / \mathrm{mL}$ for II.

Precision, Accuracy, and Recovery. The precision of the method was determined by calculating relative standard deviation (RSD) for QCs at three concentration levels over 3 days of validation tests (Table 1). Intra-day precision was $12 \%$ or less, and inter-day precision was $10 \%$ or less at each QC level. The accuracy of the method was between $91.0 \%$ and $109.0 \%$ at each QC level. Mean recoveries of atractylenolide I and II in rat plasma ranged from $86.2 \%$ to $96.3 \%$. The recovery of the IS $(50 \mathrm{ng} / \mathrm{mL})$ was $94.2 \%$.

Stability. Results from the auto-sampler showed that the analyte was stable under room temperature as well as freezethaw and long-term (30 days) conditions and were confirmed because the bias in concentrations were within $\pm 15 \%$ of their nominal values.

Application. The mean plasma concentration-time curve after intravenous $(5 \mathrm{mg} / \mathrm{kg}$ ) administration of atractylenolide I and II were shown in Figure 3. Primary pharmacokinetic parameters, based on non-compartment model analysis, were summarized in Table 2. There have been several literatures reported for the pharmacokinetic study of atractylenolide I or atractylenolide II after oral administration in rats $[1,7-10]$. However, the pharmacokinetic study of atractylenolide I or atractylenolide II after intravenous administration in rats was not reported.

\section{Conclusion}

In the present study, a simple, precise, and accurate UPLCMS/MS method for the quantitation of atractylenolide I and II in rat plasma was established, utilizing $50 \mu \mathrm{L}$ of plasma with an LLOQ of $1 \mathrm{ng} / \mathrm{mL}$. The UPLC-MS/MS method was successfully applied to a pharmacokinetic study of atractylenolide I and II after intravenous administration.
Acknowledgments. This study was supported by grants from the Wenzhou City Planning Commission project (2016B03), Wenzhou City Public Welfare Science and Technology Project (Y20150178, Y20160187, and Y20160540), Zhejiang Institute of pharmacy special scientific research projects (2016ZYY23).

\section{References}

1. Yan, H.; Sun, Y.; Zhang, Q.; Yang, M.; Wang, X.; Wang, Y.; Yu, Z.; Zhao, Y. J. Chromatogr. B: Anal. Technol. Biomed. Life Sci. 2015, 993-994, $86-92$.

2. Song, J.; Zheng, S. R.; Jin, Y.; Li, J. Planta. Med. 2014, 80, 187-192.

3. Chen, Q.; He, H.; Li, P.; Zhu, J.; Xiong, M. Biomed. Chromatogr. 2013, 27, 699-707.

4. Long, F.; Wang, T.; Jia, P.; Wang, H.; Qing, Y.; Xiong, T.; He, M.; Wang, X. Med. Sci. Monit. 2017, 23, 571-579.

5. Liu, H.; Zhang, G.; Huang, J.; Ma, S.; Mi, K.; Cheng, J.; Zhu, Y.; Zha, X.; Huang, W. J. Transl. Med. 2016, 14, 104.

6. Wang, A.; Xiao, Z.; Zhou, L.; Zhang, J.; Li, X.; He, Q. Pharm. Biol. 2016, $54,146-150$.

7. Li, Y.; Zhang, Y.; Wang, Z.; Zhu, J.; Tian, Y.; Chen, B. J. Pharm. Biomed. Anal. 2012, 58, 172-176.

8. Shi, Y. Y.; Guan, S. H.; Tang, R. N.; Tao, S. J.; Guo, D. A. Biomed. Chromatogr. 2012, 26, 1386-1392.

9. Ge, J.; Wang, Y. W.; Lu, X. C.; Sun, X. H.; Gong, F. J. Biomed. Chromatogr. 2007, 21, 299-303.

10. Wang, R.; Wang, G.; Hao, H.; Xie, H.; Zhang, J.; Wu, F. J. Chromatogr. B: Anal. Technol. Biomed. Life Sci. 2006, 831, 36-41.

11. Wen, C.; Wang, S.; Huang, X.; Liu, Z.; Lin, Y.; Yang, S.; Ma, J.; Zhou, Y.; Wang, X. Biomed. Chromatogr. 2015, 29, 1805-1810.

12. Wang, S.; Wu, H.; Geng, P.; Lin, Y.; Liu, Z.; Zhang, L.; Ma, J.; Zhou, Y.; Wang, X.; Wen, C. Biomed. Chromatogr. 2016, 30, 1145-1149.

13. Ma, J.; Wang, S.; Huang, X.; Geng, P.; Wen, C.; Zhou, Y.; Yu, L.; Wang, X. J. Pharm. Biomed. Anal. 2015, 111, 131-137.

14. Wen, C.; Zhang, Q.; He, Y.; Deng, M.; Wang, X.; Ma, J. Acta Chromatogr. 2015, 27, 81-91.

15. Ma, J.; Wang, S.; Huang, X.; Geng, P.; Wen, C.; Zhou, Y.; Yu, L.; Wang, X. J. Chromatogr. B: Anal. Technol. Biomed. Life Sci. 2015, 990, 131-137.

16. Ma, J.; Wang, S.; Zhang, M.; Zhang, Q.; Zhou, Y.; Lin, C.; Lin, G.; Wang, X. Biomed. Chromatogr. 2015, 29, 1203-1212.

17. Wang, X.; Wang, S.; Ma, J.; Ye, T.; Lu, M.; Fan, M.; Deng, M.; Hu, L.; Gao, Z. J. Pharm. Biomed. Anal. 2015, 115, 368-374.

18. Wang, X.; Wang, S.; Lin, F.; Zhang, Q.; Chen, H.; Wang, X.; Wen, C.; Ma, J.; Hu, L. J. Chromatogr. B: Anal. Technol. Biomed. Life Sci. 2015, 983-984, 125-131.

19. Wang, S.; Wu, H.; Huang, X.; Geng, P.; Wen, C.; Ma, J.; Zhou, Y.; Wang, X. J. Chromatogr. B: Anal. Technol. Biomed. Life Sci. 2015, 990, 118-124.

20. Zhang, Q.; Wen, C.; Xiang, Z.; Ma, J.; Wang, X. J. Pharm. Biomed. Anal. 2014, 90, 134-138. 